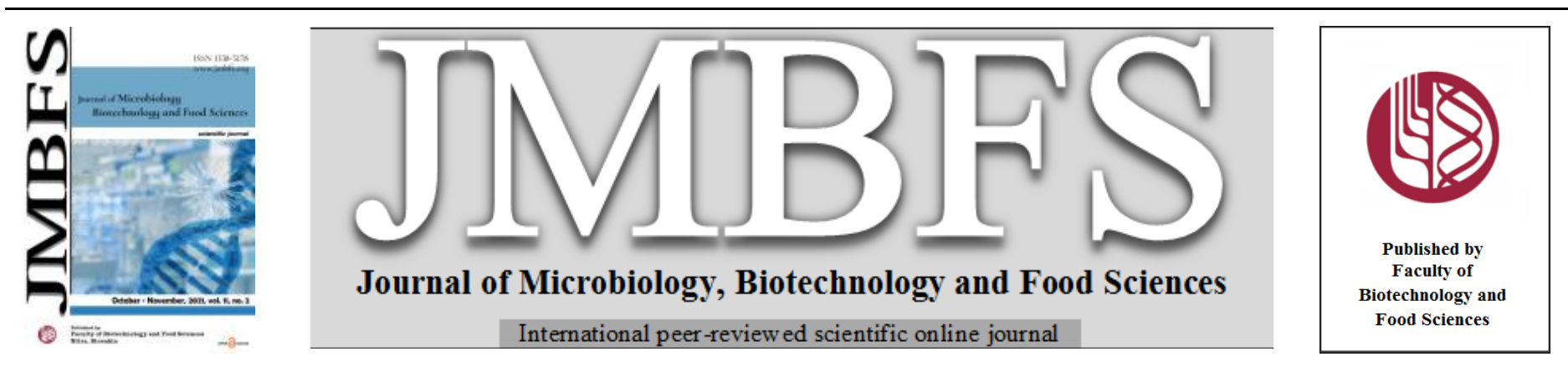

\title{
ANTITUMOR PROPERTIES OF VEGETABLE OIL EXTRACT FROM GREEN MICROALGA COELASTRELLA SP.
}

\author{
Tanya Toshkova-Yotova* ${ }^{1}$, Ani Georgieva ${ }^{2}$, Katerina Todorova ${ }^{2}$, Plamen Pilarski $^{1}$, RenetaToshkova $^{2}$
}

$\operatorname{Address}(e s)$ :

${ }^{1}$ Bulgarian Academy of Sciences, Institute of Plant Physiology and Genetics, Department Experimental algology, Acad. G. Bontchev Str., B1.23, 1113, Sofia, Bulgaria.

${ }^{2}$ Bulgarian Academy of Sciences, Institute of Experimental Morphology, Pathology and Parasitology with Museum, Department Pathology, Acad. G. Bontchev Str., B1. 25, 1113, Sofia, Bulgaria.

*Corresponding author: t toshkova_yotova@abv.bg

https://doi.org/10.15414/jmbfs.2744

\section{ARTICLE INFO}

Received 10. 3. 2020

Revised 21. 1. 2021

Accepted 16. 4. 2021

Published 1. 10. 2021

Regular article

open $\mathcal{O}$ access

\begin{abstract}
Microalgae are of great importance for the production of original natural substances of interest for food, health or biotechnological applications. Mass cultures of green microalgae are used for production of carotenoids such $\beta$-carotene, astaxanthin, canthaxanthin, lutein etc.

The aim of this study was to obtain an oil extract from a Bulgarian green microalga strain Coelastrella sp. BGV and to assess its anticancer and apoptogenic activity in vitro against human tumor cells HeLa by means of MTT and fluorescence microscopy analyses. The results showed that the oil extract obtained by a direct extraction using a common vegetable oil reduces proliferation and induces apoptosis in HeLa cells.

In contrast to widely used organic solvents for the production of carotenoids, the applied bio-solvent (sunflower oil) can be a valuable alternative approach for the needs of the food industry. The resulting oil extract showed promising antitumor and apoptosis-inducing activity against HeLa cells in vitro and potential for future use in practice.
\end{abstract}

Keywords: Coelastrella; antitumor activity, apoptosis, HeLa, sunflower oil extract

\section{INTRODUCTION}

Cancer is one of the leading causes of death in the world. In this respect cancer research has been mainly focused on the search for natural curative substances and developing a new functional food or drugs. Microalgae and seaweeds are recognized as a natural source of commercially and industrially valuable, healthpromoting and biologically active products such as carotenoid pigments, polyunsaturated fatty acids, vitamins, lipids, carbohydrates, proteins and etc. (Galasso et al., 2017; Sathasivamet al., 2019). The carotenoids astaxanthin, $\beta$ carotene, lutein, lycopene, and canthaxanthin are the major carotenoids of commercial value due to their significant roles, including: potent antioxidant properties; protection against lung, head, neck and prostate cancer; protection against neurodegenerative diseases; modulation of the immune system, growth factors and intracellular signaling pathways; regulation of cell differentiation, cell cycle and apoptosis; photoprotection against UV radiation; improvement of natural pigmentation of high-value ornamental fishes, flamingos and salmon and etc(Guedeset al., 2011; Boominathanet al., 2015; Fiedor\&Burda, 2014) Microalgal species belonging to Chlorophyta, such as Dunaliella salina, Haematococcus pluvialis, Chlorella vulgaris, Chlorella zofingiensis and Chlorella pyrenoidosa have been successfully cultivated for production of $\beta$ carotene, astaxanthin, canthaxanthin, lutein and other carotenoids (Leu et al. 2014; Zhang et al., 2014; Gong et al., 2016). The Coelastrella microalgae are also able to produce a different amount of canthaxanthin, astaxanthin and betacarotene depending on the various stress, limiting conditions and strain (Abe $\boldsymbol{e t}$ al., 2007; Iyeret al., 2015). However the production of microalgal carotenoids is not yet sufficiently cost-effective and many challenges still exist in processing (Poojary et al., 2016; Saini et al., 2018). In the last years, there has been an increased interest towards the production of natural carotenoids using nontoxic solvents in terms of environmental safety and also its application in foods, nutriceuticals, medicines, cosmetics and etc. (Krichnavaruk et al., 2008; Wang et al., 2012). Sunflower oil is reported to be a better choice (Chen et al., 2016).

The aim of the present study was to prepare an oil extract from a Bulgarian green microalgal strain Coelastrella $\mathrm{sp}$. BGV and to investigate its in vitro antitumor activity against human cervical carcinoma cells HeLa.

\section{MATERIAL AND METHODS}

\section{Algal material and culture conditions}

The green microalga, Coelastrella sp. BGV, was isolated from a stagnant water in metal trough near Varvara village, Bulgaria (Dimitrova, 2016; Dimitrova, 2018). The microalga was cultured and maintained as non-axenic monoculture in Šetlik medium modified by Georgievet al., (1978) at $28^{\circ} \mathrm{C}$ under continuous unilateral illumination by cool-white fluorescent lamps at a photon flux density of $132 \mu \mathrm{mol} \mathrm{m} \mathrm{m}^{-2}$. Batch cultures were bubbled with air $\left(2-3 \% \mathrm{CO}_{2} ; \mathrm{v} / \mathrm{v}\right)$ in 200 $\mathrm{ml}$ flat glass bottles.

\section{Oil Extraction}

For direct extraction of carotenoids (astaxanthin) according to method of Kang $\boldsymbol{e}$ $\boldsymbol{a l . , ( 2 0 0 8 )}$ ), the algal suspension (from stationary phase $-10^{\text {th }}$ day) was mixed with sunflower oil in a 1: 1 ratio. A relatively long extraction time (48 h) was used in order to achieve complete extraction of the substances under continuous stirring. All procedures were performed at room temperature. After vigorous stirring, the mixture was allowed to settle under gravity into a separating funnel. The bottom layer is separated after delamination. The top layer was centrifuged at $4000 \mathrm{rpm}$ for $10 \mathrm{~min}$ and the amount of pigment (astaxanthin) was measured spectrophotometrically at $480 \mathrm{~nm}$. The extract was collected in glass tubes.

Anti-Cancer Potential. The oil extract (OE) of the cells of Coelastrella sp. BGV was tested for its antiproliferative and apoptosis-inducing potential towards HeLa cervical cancer cells by MTT assay and florescence microscopic analyzes.

\section{Cell line and culture conditions}

The HeLa cell line from the Culture Collection of the IEMPAM-Institute, BAS was used. The cells were cultivated in Dulbecco's modified Eagle's medium (DMEM; Gibco/Invitrogen) with $10 \%$ Fetal Bovine Serum (FBS) in $37^{\circ} \mathrm{C}$ humidified incubator containing $5 \% \mathrm{CO}_{2}$.

\section{MTT assay}

Cell viability was measured with the 3-(4,5-dimethylthiazol-2-yl)-2, 5dipheniltetrazolium bromide (MTT) assay (Mosmann, 1983) after 48 hours of 
treatment. For this purpose, HeLa cells in a logarithmic phase of growth were adjusted to $1 \times 10^{5}$ cells $/ \mathrm{ml}$ and $100 \mu 1$ aliquots were transferred to 96 -well plates. Different concentration $(31.3 ; 62.5 ; 125 ; 250 ; 500$ and $1000 \mu \mathrm{g} / \mathrm{mL})$ of the $\mathrm{OE}$ were added to each well after $24 \mathrm{~h}$. Untreated tumor cells used as negative controls. The absorbance was measured with a microplate ELISA reader (TECAN, Sunrise TM, Groedig/Salzburg, Austria) at $570 \mathrm{~nm}$ reference wavelength. The effect of tested microalgal OE was expressed as the percent of the cell viability of the untreated control cells.

\section{Morphological investigations by fluorescent microscopy}

HeLa tumor cells were seeded at an optimal density of $0.5 \times 10^{5} / \mathrm{ml}$ on glass slides placed in the bottom of 24-well plates and grown overnight in $\mathrm{CO}_{2}$ incubator under standard conditions (complete DMEM medium containing $10 \%$ fetal bovine serum). The cell were treated with $\mathrm{OE}$ at a concentration equal to the half of the maximal concentration used in the MTT assay. After an additional incubation for 24 hours, the glass lamellas were washed in PBS and stained by two methods - the acridine orange (AO)/ethidium bromide (EB) staining according to Wahab et al., (2009) and DAPI staining based on the established protocol. Stained cells were mounted with glycerol on microscope slides and analyzed by a fluorescent microscope (Leica DM 500B, Wetzlar, Germany).

\section{Statistical Analysis}

The experimental results are presented as the mean values and their standard deviations ( \pm S.D.). One-way analysis of variance (ANOVA) followed by Bonferroni's post hoc test was performed using GraphPad PRISM software, Version 5 (GraphPad Prism Software Inc., San Diego, USA). Values of * $p<0.05$ were considered to indicate statistical significance.

\section{RESULTS}

\section{Preparation and characterization of oil extract from Coelastrella sp. BGV}

Sunflower oil extract from the Coelastrella sp. BGV microalga was prepared. The absorption spectrum of the extract showed a major absorbance in the $400-500 \mathrm{~nm}$ range with maximum at $460 \mathrm{~nm}$, which is an indication of presence of carotrenoid (astaxanthin) pigment in the oil (Fig. 1).

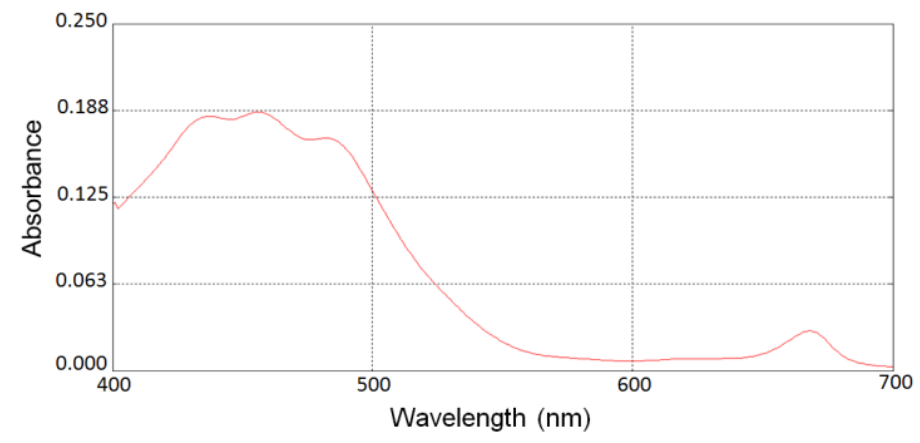

Figure 1 Absorption spectra of the sunflower oil extract from Coelastrellasp. BGV cells. The extract sample was diluted twenty-fold before measuring the absorbance

\section{Effect of the oil extract from Coelastrella sp. BGV on the tumor cell viability}

The effect of OE on HeLa cell proliferation was determined by MTT assay after $24 \mathrm{~h}$ incubation with OE (Fig. 2).

\section{Apoptogenic effect of the oil extract from Coelastrella sp. BGV}

The morphology of the treated HeLa cells was examined under a fluorescent microscope. Consistent with the MTT assay, significant cell morphology alterations as well as nuclear changes were detected in the tumor cells, which suggested that the cell death occurred via cellular apoptosis (Fig. 3).

The untreated HeLa cells showed a typical uniform monolayer growth and morphology characteristic for the corresponding cell type. The cells were slightly elongated in shape, greenish-colored with pale green nuclei, containing 3-4 yellow-green nucleolus and clusters of yellow-orange granules located in the perinuclear region (Fig. 3, a). HeLa tumor cells cultured $24 \mathrm{~h}$ in the presence of OE (Fig. 3, b) showed a pronounced cytopathic alterations. Monolayer growth was impaired and the cells exhibited morphological changes characteristic of early (rounded cells with unequally distributed nuclear chromatin) and late apoptosis (cells with a bright orange nucleus with chromatin condensation and cell membrane blebings). What makes an impression was the presence of cells reflecting the gradual transition from early to late apoptosis (Fig. 3,b). HeLa cells cultured in the presence of Doxorubicin (positive control) were mainly with late apoptotic changes - saturated orange-red stained nuclei with condensation and fragmentation of the chromatin (Fig.3,c).

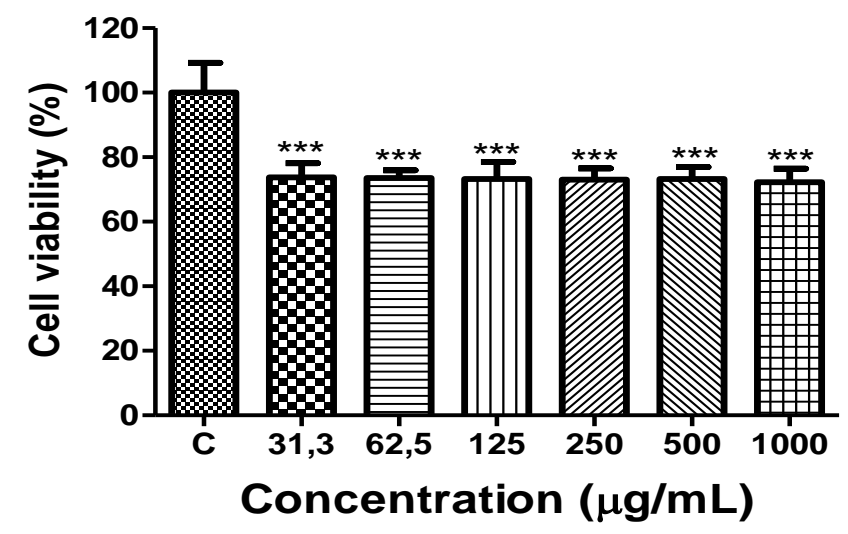

Figure 2 Effect of oil extract (OE) of Coelastrella sp. BGV on the proliferation of HeLa tumor cells determined with MTT assay at the 24th h. *** $(\mathrm{p}<0.001)$

As shown at Fig. 2, the oil extract of green microalga Coelastrella sp. BGV decreased significantly the proliferation of the HeLa tumor cells, to values of 72 $73 \%$.

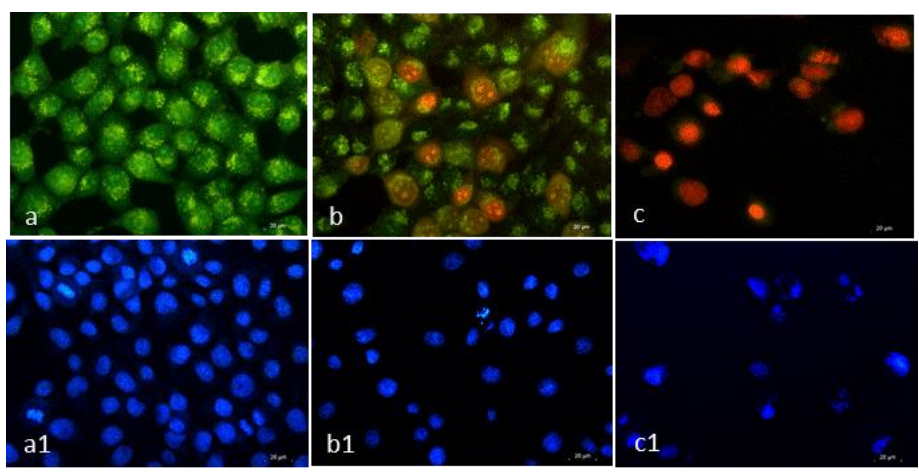

Figure 3 Fluorescence microscopy of HeLa tumor cells cultured for 24 hours in presence of Coelastrella sp. BGV oil extract. Legend: a,a1 - HeLa in culture medium (control); b,b1- HeLa in the presence of OE, c,c1- HeLa in the presence of Doxorubicin. $\mathrm{AO} / \mathrm{EtBr}$ (upper row) and DAPI (bottom row) staining. Objective: $40 \mathrm{X}$

Nuclear changes in the HeLa cells treated for 24 hours with OE from Coelastrella sp. BGV were analyzed after staining with DAPI (Figure 3, bottom line). The untreated control HeLa cells (Fig 3, a1) were with intact nuclei, round to slightly oval in shape with smooth edges and evenly distributed chromatin. Cell nuclei at different phases of mitosis were observed. In contrast, OE-treated cells exhibited atypical nuclear morphology - a pronounced nuclear polymorphism, unequal outline of nuclei, irregularly distributed chromatin (condensation and marginalization), nucleus fragmentation and apoptotic body formation (Fig 3, b1). HeLa tumor cells treated with the anthracycline anti-tumor antibiotic Doxorubicin were used as a positive control (Fig 3, C1). A heavily reduced number of nuclei with unequal outlines and condensed chromatin, as well as fragmented nuclei and apoptotic bodies were observed.

\section{DISSCUSSION}

Throughout the world, there is growing interest in health foods and medicines containing natural bioactive substances such as carotenoids, antioxidants, vitamins, proteins etc. Microalgae offer great potential as a source of natural compounds with desirable properties that are receiving attention in the food, pharmaceutical, cosmetic industries etc. (Galasso et al., 2017; Sathasivam et al., 2019). Classical organic solvent extraction techniques are widespread technologies for extraction of lipids and pigments, but usually imply the use of large amounts of solvents, and the risk of thermal denaturation or transformation of molecules of interest. In the last years, there has been an increased interest in using extraction techniques involving nontoxic solvents (vegetable oils) (Krichnavaruk et al., 2008; Wang et al., 2012). In the present study, we used direct extraction method with vegetable oil to prepare sunflower oil extract rich in carotenoids. The spectrophotometric analysis showed an absorption spectrum of $\mathrm{OE}$ in the carotenoid-specific region. Similar results were reported by Kang et al. (2008), who identified astaxanthin from Haematococcus culture using vegetable oils at $474 \mathrm{~nm}$. The presence of a small peak in the range of $700 \mathrm{~nm}$ is most likely due to the other type of oil-soluble pigments contained in the present 
extract, for example chlorophyll. The advantage of using vegetable oils is that they are considered a good barrier against oxygen, slowing oxidation processes, in addition to being used in the subsequent application in foods (Rao et al., 2007).Abe et al. (2007) showed that the algal extract from Coelastrella striolate var. multistriata containing the carotenoids-canthaxanthin, astaxanthin and $\beta$ carotene had an antioxidant potential in lipid foods. In addition to the antioxidant properties, carotenoids exhibit anti-inflammatory, antiproliferative, neuroprotective activities, improve insulin signals, and reduce lipid levels (Fiedoret al., 2014; Gong et al., 2016). Regulation of the cell differentiation, cell cycle and induction of apoptosis were also reported (Niranjana et al., 2015; Li et al., 2015). Our study of the anticancer potential of OE from Coelastrella $\mathrm{sp}$ BGV showed antiproliferative activity and induction of apoptosis in HeLa tumor cells in vitro. Inhibition of the cell proliferation of colon cancer cells by Neochloriso leoabundans carotenoids was observed by Castro-Puyanaet al., (2017). Recent studies pointed that the antitumor activity of astaxanthin may be exerted through several different mechanisms of action and influence on a multitude of molecular and cellular processes (Zhanget al., 2015). The antiproliferative effect of astaxanthin against CBRH-7919 (human hepatoma), SHZ 88 (rat breast), Lewis (mouse lung) cells and human HCT-116 colon cancer cells (Song et al., 2011; Palozza et al., 2011) was reported. The results obtained by Song et al., (2011; 2012) and Kavithaet al., (2013) suggests that astaxanthin could induce mitochondria-mediated apoptosis in the cancer cells. The mechanisms that mediate the anticancer action of astaxanthin have not yet been fully elucidated, but a number of molecular targets [c-Jun N-terminal kinase (JNK), nuclear factor kappa $(\mathrm{NF}-\kappa \mathrm{B})$; STAT-3, etc] have been proposed (Niranjana et al., 2015; Li et al., 2015; Zhang et al., 2015). Targeting these molecular targets may result in apoptosis of cancer cells.target specific molecules involved in the growth and spread of cancer cells

\section{CONCLUSION}

A direct extraction of carotenoid (astaxanthin) from the Coelastrella sp. BGV microalga using sunflower oil as a solvent was reported. The proposed method reduces the use of classic organic solvents, allows the use of renewable natural product, and ensures a safe and high-quality extract/product. In this way, there is no need for subsequent separation of oil and carotenoids, since the oil rich in carotenoids can be used directly in different products. In addition, we have shown for the first time that the sunflower oil extract from the Coelastrella sp. BGV microalga has antiproliferative activity and induces apoptotic cell death in HeLa cancer cells in vitro. In order to determine whether the OE induced apoptosis in HeLa cells, we used double fluorescent AO/PI and DAPI staining. The results showed that after OE treatment for $24 \mathrm{~h}$, the number of early and late apoptotic cells was significantly higher compared with the control. As well as the changed DNA located in apoptotic nuclei was easily stained with DAPI reagent and showed bright blue fluorescence. The results were consistent with those obtained by MTT test. The present study showed a promising anti-tumor activity of the $\mathrm{OE}$, however further research is needed to identify the responsible bioactive components of microalgae oil extract and to elucidate the molecular mechanisms of their antineoplastic effects.

\section{REFERENCES}

Abe, K., Hattori, H., \& Hirano, M. (2007). Accumulation and antioxidant activity of secondary carotenoids in the aerial microalga Coelastrella striolata var. $\begin{array}{llll}\text { multistriata. Food chemistry, } & \text { 100(2), }\end{array}$ https://doi.org/10.1016/j.foodchem.2005.10.026

Boominathan, M., \& Mahesh, A. (2015). Seaweed carotenoids for cancer therapeutics. In Handbook of Anticancer Drugs from Marine Origin (pp. 185203). Springer, Cham. https://doi.org/10.1007/978-3-319-07145-9_10

Castro-Puyana, M., Pérez-Sánchez, A., Valdés, A., Ibrahim, O. H. M., SuarezÁlvarez, S., Ferragut, J. A., \& García-Cañas, V. (2017). Pressurized liquid extraction of Neochloris oleoabundans for the recovery of bioactive carotenoids with anti-proliferative activity against human colon cancer cells. Food Research International, 99, 1048-1055. https://doi.org/10.1016/j.foodres.2016.05.021

Chen, C. Y., Hsieh, C., Lee, D. J., Chang, C. H., \& Chang, J. S. (2016) Production, extraction and stabilization of lutein from microalga Chlorella sorokiniana MB-1. Bioresource technology, 200, 500-505. https://doi.org/10.1016/j.biortech.2015.10.071

Dimitrova P., Marinova G. \& Pilarski P. (2016). Preliminary studies on the growth and biochemical composition of a promising carotenoid strain Coelastrella sp. Science \& Technologies 6(3), 71-78.

Dimitrova P. H., Stoyneva-Gärtner M. P., Uzunov B. A., Gärtner G. (2018) Review of the algological studies of Bulgarian Black Sea coastal water bodies (1890-2017) with special attention to the newly described and threatened species. Acta zoologica bulgarica, Suppl. 11, 27-42. Fiedor, J., \& Burda, K. (2014) Potential role of carotenoids as antioxidants in human health and disease. Nutrients, 6(2), 466-488. https://doi.org/10.3390/nu6020466
Galasso, C., Corinaldesi, C., \& Sansone, C. (2017). Carotenoids from marine organisms: Biological functions and industrial applications. Antioxidants, 6(4), 96. https://doi.org/10.3390/antiox6040096

Georgiev, D., Dilov H., \& Avramova S.(1978). Millieu nutritif tamponne et méthode de culture intensive des microalgues vertes. Hydrobiology, 7, 14-23.

Gong, M., \& Bassi, A. (2016). Carotenoids from microalgae: A review of recent developments. Biotechnology Advances, 34(8), 1396-1412.

Guedes, A. C., Amaro, H. M., \& Malcata, F. X. (2011). Microalgae as sources of high added-value compounds - a brief review of recent work. Biotechnology progress, 27(3), 597-613. https://doi.org/10.1016/j.biotechadv.2016.10.005

Iyer, G., Nagle, V., Gupte, Y. V., Desai, S., Iyer, M., Moramkar, N., \& Sawant, V. (2015). Characterization of high carotenoid producing Coelastrella oocystiformis and its anti-cancer potential. International J. of Current Microbiology and Applied Sciences, 4(10), 527-536.

Kang, C. D., \& Sim, S. J. (2008). Direct extraction of astaxanthin from Haematococcus culture using vegetable oils. Biotechnology letters, 30(3), 441444. https://doi.org/10.1007/s10529-007-9578-0

Kavitha, K., Kowshik, J., Kishore, T. K. K., Baba, A. B., \& Nagini, S. (2013) Astaxanthin inhibits $\mathrm{NF}-\kappa \mathrm{B}$ and $\mathrm{Wnt} / \beta$-catenin signaling pathways via inactivation of Erk/MAPK and PI3K/Akt to induce intrinsic apoptosis in a hamster model of oral cancer. Biochimica et Biophysica Acta (BBA)-General Subjects, 1830(10), 4433-4444. https://doi.org/10.1016/j.bbagen.2013.05.032

Krichnavaruk, S., Shotipruk, A., Goto, M., \& Pavasant, P. (2008). Supercritical carbon dioxide extraction of astaxanthin from Haematococcus pluvialis with vegetable oils as co-solvent. Bioresource technology, 99(13), 5556-5560. https://doi.org/10.1016/j.biortech.2007.10.049

Leu, S., \& Boussiba, S. (2014). Advances in the production of high-value products by microalgae. Industrial Biotechnology, 10(3), 169-183. https://doi.org/10.1089/ind.2013.0039

Li, J., Dai, W., Xia, Y., Chen, K., Li, S., Liu, T., \& Yin, Q. (2015). Astaxanthin inhibits proliferation and induces apoptosis of human hepatocellular carcinoma

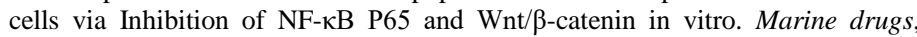
13(10), 6064-6081. https://doi.org/10.3390/md13106064

Mosmann, T. (1983). Rapid colorimetric assay for cellular growth and survival: application to proliferation and cytotoxicity assays. Journal of immunological methods, 65(1-2), 55-63. https://doi.org/10.1016/0022-1759(83)90303-4

Niranjana, R., Gayathri, R., Mol, S. N., Sugawara, T., Hirata, T., Miyashita, K., \& Ganesan, P. (2015). Carotenoids modulate the hallmarks of cancer cells. Journal of Functional Foods, 18, 968-985. https://doi.org/10.1016/j.jff.2014.10.017

Palozza, P., Torelli, C., Boninsegna, A., Simone, R., Catalano, A., Mele, M. C., \& Picci, N. (2009). Growth-inhibitory effects of the astaxanthin-rich alga Haematococcus pluvialis in human colon cancer cells. Cancer Letters, 283(1), 108-117. https://doi.org/10.1016/j.canlet.2009.03.031

Poojary, M. M., Barba, F. J., Aliakbarian, B., Donsì, F., Pataro, G., Dias, D. A., \& Juliano, P. (2016). Innovative alternative technologies to extract carotenoids from microalgae and seaweeds. Marine drugs, 14(11), 214 https://doi.org/10.3390/md14110214

Rao, A. R., Sarada, R., \& Ravishankar, G. A. (2007). Stabilization of astaxanthin in edible oils and its use as an antioxidant. Journal of the Science of Food and Agriculture, 87(6), 957-965. https://doi.org/10.1002/jsfa.2766

Saini, R. K., \& Keum, Y. S. (2018). Carotenoid extraction methods: A review of recent developments. Food Chemistry, 240, 90-103. https://doi.org/10.1016/j.foodchem.2017.07.099

Sathasivam, R., Radhakrishnan, R., Hashem, A., \& Abd Allah, E. F. (2019). Microalgae metabolites: A rich source for food and medicine. Saudi journal of biological sciences, 26(4), 709-722. https://doi.org/10.1016/j.sjbs.2017.11.003

Song, X. D., Zhang, J. J., Wang, M. R., Liu, W. B., Gu, X. B., \& Lv, C. J. (2011) Astaxanthin induces mitochondria-mediated apoptosis in rat hepatocellular carcinoma CBRH-7919 cells. Biological and Pharmaceutical Bulletin, 34(6), 839-844. https://doi.org/10.1248/bpb.34.839

Song, X., Wang, M., Zhang, L., Zhang, J., Wang, X., Liu, W., ... \& Lv, C. (2012). Changes in cell ultrastructure and inhibition of JAK1/STAT3 signaling pathway in CBRH-7919 cells with astaxanthin. Toxicology mechanisms and methods, 22(9), 679-686. https://doi.org/10.3109/15376516.2012.717119

Wahab, A., S. I., Abdul, A. B., Alzubairi, A. S., Mohamed Elhassan, M., \& Mohan, S. (2009). In vitro ultramorphological assessment of apoptosis induced by zerumbone on (HeLa). BioMed Research International, 2009. https://doi.org/10.1155/2009/769568

Wang, L., Yang, B., Yan, B., \& Yao, X. (2012). Supercritical fluid extraction of astaxanthin from Haematococcus pluvialis and its antioxidant potential in sunflower oil. Innovative Food Science \& Emerging Technologies, 13, 120-127. https://doi.org/10.1016/j.ifset.2011.09.004

Zhang, J., Sun, Z., Sun, P., Chen, T., \& Chen, F. (2014). Microalgal carotenoids: beneficial effects and potential in human health. Food \& function, 5(3), 413-425. https://doi.org/10.1039/c3fo60607d

Zhang, L., \& Wang, H. (2015). Multiple mechanisms of anti-cancer effects exerted by astaxanthin. Marine drugs, 13(7), 4310-4330. https://doi.org/10.3390/md13074310 